\title{
Augmenting Noun Taxonomies by Combining Lexical Similarity Metrics
}

\author{
Mark Stevenson \\ Reuters Ltd \\ 85 Fleet Street \\ London \\ EC4P 4AJ \\ mark.stevenson@reuters.com
}

\begin{abstract}
This paper presents a method for augmenting taxonomies with domain information using a simple combination of three existing lexical similarity metrics. The combined approach is evaluated by comparing their results against the annotated SEMCOR corpus. An implementation is described in which WordNet is augmented with thesaural information from the CIDE+ machine readable dictionary.
\end{abstract}

\section{The Tennis Problem}

Lexical taxonomies, in particular WordNet (Fellbaum, 1998), are now widely used in NLP for applications including semantic tagging, text categorisation and parsing (Harabagiu and Chai, 1998). WordNet consists of sets of lexical items (words and phrases) with similar meanings called synsets which are organised into a hyponomy (IS_A) hierarchy. For example, "doctor" and "physician" are in the same synset which is directly subsumed by "medical practitioner". However the coverage of lexical semantics in WordNet is not comprehensive, items such as discourse information are not included. For example, "tennis player" (a hyponym of person) is not closely related to "racket", "balls" or "net" (hyponyms of artifact). Motivated by this example, Fellbaum (1998) dubbed this the "tennis problem". Taxonomies omitting this information are ignoring potentially valuable information which could be helpful for applications such as information retrieval (IR), word sense disambiguation, information extraction and parsing.

This paper reports a step towards a solution for the tennis problem by adding thesaural relations to the noun taxonomy in WordNet (version 1.6). The aim is to produce groups of noun synsets which are related by topic or do- main. Once identified these links can be added to WordNet to denote this new form of lexical information, which is in addition to the existing hyponomy and hypernymy relations.

An example fragment of the WordNet hierarchy with the types of links we aim to add is shown in Figure 1. The existing relations, shown as unbroken and dashed lines, demonstrate the relative distance between items such as "ball boy" and "tennis ball". This link would be made explicit by the addition of the thesaural links which are shown as dotted lines.

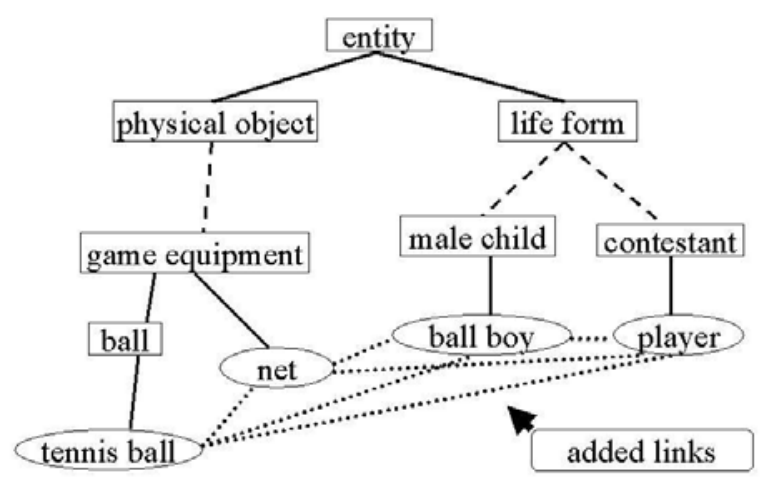

Figure 1: Fragment of WordNet hierarchy with proposed new links shown as dotted lines.

This new information is obtained from the related words classes found in the CIDE+ machine readable dictionary (Procter, 1995). The main problem with using these existing classes is that it is not clear which of the possible synsets in WordNet each word refers to since they are not disambiguated against WordNet. Therefore the problem reduces to disambiguating sets of related nouns against the senses in the taxonomy. This problem is addressed by 
combining existing disambiguation techniques and applying this approach to WordNet and CIDE+ (Section 2). An evaluation of this approach is presented in Section 3. Some related work is described in Section 4.

\section{Disambiguating Thesaural Classes}

The machine readable version of the CIDE+ dictionary contains thesaural information in the form of 1924 related word classes. Example categories are Board Games (dice_1_0, square_1_3, shake_1_12, backgammon_1_0 ...) and Colours (silver_1_1, redness_1_0, black_1_22, amber_1_0 ...). Each member of the category relates to a particular CIDE+ sense which allows access to the textual definitions contained in the dictionary. This extra information is the main advantage of using CIDE+ related word categories compared to a traditional thesaurus which consists only of groups of related words untagged for sense.

The first stage is to disambiguate each noun in CIDE+ thesaurus classes against WordNet. Formally, assume that CIDE+ thesaurus class, $N$, contains the word $W$ with senses $\left\{s_{1}, s_{2} \ldots s_{n}\right\}$. We wish to assign a value to each possible WordNet sense for $W$ indicating the likelihood of that sense being relevant given that $W$ is a member of the noun group $N$. This is achieved using a combination of existing lexical similarity metrics which are now described.

\subsection{Information and semantic similarity}

Resnik (1999) reports an algorithm for disambiguating groups of nouns against their WordNet synsets. This is achieved by assigning each node in WordNet a numerical value which indicates its information content. This value is derived from frequency of occurrence in a training corpus by assuming that each parent of a term is implied by a corpus instance. The actual information content value is calculated, in the standard information theoretic way, as the negative logarithm of the probability. When these values have been calculated each node in the taxonomy is more informative than its parents, for example, "nurse" and "professor" are each more informative than "professional". Disambiguation is carried out by comparing senses in a pairwise fashion and selecting the combination which yields the highest information value.
Resnik (1999) trained his algorithm on the 1 million word Brown Corpus of American English (Francis and Kucera, 1982). Our reimplementation used the written portion of the British National Corpus (Burnard, 1995) which contains roughly 90 million words. However this additional training data did not seem to make much difference to the algorithm's performance.

More formally, Resnik's algorithm returns a value, $\phi\left(s_{i}\right)$, for each each sense, $s_{i}$, associated with a word $W$ which is a member of the CIDE+ noun group $N$ indicating the likelihood that the sense is relevant to the noun group. We normalise these values for each sense thus to create the Information Content $(I C)$ metric:

$$
I C_{i}=\frac{\phi\left(s_{i}\right)}{\sum_{j=1}^{n} \phi\left(s_{j}\right)}
$$

\subsection{Conceptual Distance}

In Resnik's disambiguation method distance between senses in the taxonomy is determined by their information content in the WordNet hierarchy. Agirre and Rigau (1996) presented a method for disambiguating nouns in text where the distance between WordNet senses is determined solely by the structure of the taxonomy, i.e. the corpus frequencies used by Resnik were not considered. This method is known as Conceptual Distance. It prefers senses closely related in the WordNet hierarchy taking into account the depth of the hierarchy, density of senses and length of shortest path between concepts. Like Resnik's method, this approach takes a set of nouns as input and calculated the Conceptual Distance value for each possible WordNet sense relative to the words in the set.

Assuming that the value returned by the conceptual distance algorithm for sense $s_{i}$ is $\theta\left(s_{i}\right)$ then the value given by the conceptual distance $(C D)$ metric for a sense is given by:

$$
C D_{i}=\frac{\theta\left(s_{i}\right)}{\sum_{j=1}^{n} \theta\left(s_{j}\right)}
$$

The software which implements this disambiguation algorithm has been made publically available and was used for the experiments described in this paper. 


\subsection{Word Overlap Metric}

The Information Content and Conceptual Distance metrics derive their disambiguation information from the structure of WordNet's taxonomy and corpus information. Lesk (1986) suggested an alternative approach in which similarity is defined as the number of content words shared by the textual definitions of senses.

This method is used to identify the WordNet synset which is most similar to a CIDE+ sense by comparing the textual definition of that sense against the textual definition of all potential WordNet synsets. We call this metric the Dictionary Overlap (DO) measure and, like the previous pair, the value it returns is normalised against the total sum of word matches across all senses. So if we define $\alpha_{i}$ as the number of overlapping words in CIDE+ definition and gloss of the sense $s_{i}$ then $D O$ is calculated in our implementation thus:

$$
D O_{i}=\frac{\alpha\left(s_{i}\right)}{\sum_{j=1}^{n} \alpha\left(s_{j}\right)}
$$

This metric can be implemented more easily than the previous pair. Our implementation preprocesses the definitions in WordNet and CIDE+ by removing stop words and empty heads. The remaining words are reduced to their morphological roots.

\subsection{Combining Metrics}

So far we have described three metrics which could be used to disambiguate the sense in CIDE+ noun groups against WordNet synsets. An obvious next step would be to combine them. This is done by computing the sum and product of various combinations shown in Table 1. The combinations of metrics are computed from the value of the relevant combination for a particular sense normalised by the total value for that combination across all possible senses. Thus, for example, the value of the $I C+C D$ metric combination for sense $i$ is given by:

$$
(I C+C D)_{i}=\frac{\phi\left(s_{i}\right)+\theta\left(s_{i}\right)}{\sum_{j=1}^{n}\left(\phi\left(s_{j}\right)+\theta\left(s_{j}\right)\right)}
$$

\section{Evaluation}

To evaluate the disambiguation method we require some resource which lists the appropriate

\begin{tabular}{|rl|}
\hline \multirow{3}{*}{ Single metric } & $\mathbf{1} D O$ \\
& $\mathbf{2} I C$ \\
& $\mathbf{3} C D$ \\
\hline & $\mathbf{4} D O+I C$ \\
$\mathbf{5} D O+C D$ \\
$\mathbf{6} I C+C D$ \\
& $\mathbf{7} D O+I C+C D$ \\
\hline & $\mathbf{8} D O * I C$ \\
$\mathbf{9} D O * C D$ \\
Product & $\mathbf{1 0} I C * C D$ \\
& $\mathbf{1 1} D O * I C * C D$ \\
\hline
\end{tabular}

Table 1: Combination of metrics

WordNet sense for each of the members of the CIDE+ categories. To our knowledge no such resource exists and we are forced to adapt an existing resource. The most widely used and reliable text tagged with WordNet senses is SEMCOR (Landes et al., 1998), a 200,000 word portion of the Brown corpus semantically tagged as part of the WordNet project. ${ }^{1}$ This corpus consists of 103 files on a wide variety of topics, each concerned with a particular subject. Content words are tagged with WordNet synset numbers but there is no direct way of telling which of these are related to CIDE+ senses. However, when semantically related nouns occur together they do so with the related meanings. For example, if "monitor", "drive", "zip" and "screen" occur in the same text it is highly likely that they are all used with their senses related to computer hardware. In addition nouns appearing in a text on a given topic are likely to be related to that topic. So the word "Java" is more likely to mean 'programming language' than 'coffee' or 'island' in a text about software development. These two observations about the behaviour of noun meanings can be used to automatically derive disambiguated noun groups suitable for our evaluation from the SEMCOR texts. It is likely that in SEMCOR texts which contain a large portion of the types from a particular thesaurus class those words will be used with the WordNet sense appropriate to that class.

\footnotetext{
${ }^{1}$ The semantic tags in SEMCOR refer to WordNet version 1.5 although the release now contains a mapping for nouns between WordNet versions 1.5 and 1.6 which was used to adapt the corpus to use the lexicon in these experiments.
} 
To match SEMCOR texts against CIDE+ categories we compared each text against each category and attached a score to their relation based on the percentage of words in the category which appeared at least once in the document. This allowed us to produce a ranked list of document-category pairs based on this simple measure of relatedness. Any documents in which a CIDE+ category's words were used with less than 5 tokens were discarded. We then extracted the 10 highest ranking pairs, ignoring any categories which have already appeared. In effect this is a naive information retrieval (IR) system in which SEMCOR is the document collection and the words in the CIDE+ related word categories are the queries. We did not use a full IR system as the results from this simple method appeared adequate for the evaluation.

Table 2 shows the ten CIDE+ categories used in our evaluation and the document associated with each. The first column shows the CIDE+ category number and description, the second the percentage of types in that category followed by the number of types and tokens from the category which appear in the SEMCOR document listed in the next column. The final column contains a short description of the topic for the SEMCOR file. A first observation is that there is a reasonable semantic relation between each of the CIDE+ categories and the texts to which they were mapped.

A distinct advantage of this approach is that it allows more than one sense in WordNet to be associated with a CIDE+ sense. This is necessary since the lexicographers for each resource may have made different decisions about how rough or fine grained the sense distinctions should be. Table 3 lists the words used with more than one sense in each of the remaining four SEMCOR files. A first observation is that all senses appear consistent with the CIDE+ category related to that file (listed in Table 2). The two senses of "surface" are extremely similar while "football" and "church" exhibit clear regular polysemy. This analysis is consistent with the results reported by both Gale et al. (1992) and Krovetz (1998). The first claimed that most words are used with the same broad meaning (or homograph) in a given discourse while Krovetz claimed that closely related senses are often observed in the same dis- course.

\subsection{Evaluation Metric}

Some measure is required to compare the system output with the senses found in the SEMCOR files. Our system does not return a single sense, instead it assigns a score to each which can be viewed as a probability distribution describing the likelihood of each synset belonging to the class in question. We also know that some of the SEMCOR files contain words which are used in more than one sense and consequently their sense taggings can also be viewed as a probability distribution. Resnik and Yarowsky (1997) proposed the cross entropy metric for comparing a probability distribution produced by a disambiguation technique with disambiguated text. It is calculated according to the following formula:

$$
C E(t(x), s(x))=-\sum_{x \in S} t(x) \log _{2} s(x)
$$

where $S$ is the set of senses for the word in question, $t(x)$ is the probability distribution for those senses observed in the SEMCOR files and $s(x)$ the probability distribution obtained from our system. A perfect match between the distributions returns their entropy (which are equal). Higher values indicate lower agreement between the distributions.

To compare the output of our system for all words in a thesaurus class against the distribution found in a SEMCOR document we devised the Average Cross Entropy (ACE) metric:

$$
\mathrm{ACE}=\frac{\sum_{w \in W} C E(t(w), s(w))}{|W|}
$$

where $W$ is the set of word types occurring in the document and $t(w)$ and $s(w)$ are the relevant probability density functions. This compares the distribution of each word appearing in the text with that output by the system and adds extra weight for words which appear frequently in the SEMCOR text.

The theoretical minimum for this measure, calculated by assuming a perfect match between the two distributions was found to be 0.07 across all 10 SEMCOR texts. The average entropy for the texts other than those mentioned in Table 


\begin{tabular}{|c|c|c|c|c|}
\hline CIDE+ Category & Overlap & $\begin{array}{l}\text { Types/ } \\
\text { tokens }\end{array}$ & File & Description of text topic \\
\hline 268 Names of months & 0.77 & $8 / 11$ & $b r-j 56$ & $\begin{array}{l}\text { history of utilities in US town } \\
\text { (mentions months several times) }\end{array}$ \\
\hline 325 Planets & 0.73 & $6 / 44$ & br-j01 & astronomy \\
\hline 1528 Bays and gulfs & 0.67 & $4 / 7$ & $\mathrm{br}-\mathrm{k} 16$ & $\begin{array}{l}\text { portion of novel (frequent men- } \\
\text { tions of geographical features) }\end{array}$ \\
\hline $\begin{array}{l}1253 \text { Atoms, molecules and } \\
\text { sub-atomic particles }\end{array}$ & 0.67 & $3 / 17$ & br-j04 & sub-atomic chemistry \\
\hline 373 Secondary education & 0.54 & $2 / 7$ & br-a02 & US senate debates on education \\
\hline 486 Poultry & 0.53 & $6 / 25$ & br-k27 & $\begin{array}{l}\text { portion of novel (describes char- } \\
\text { acter's hens) }\end{array}$ \\
\hline $\begin{array}{l}147 \text { Extrasensory percep- } \\
\text { tion, Telepathy, psychics }\end{array}$ & 0.5 & $3 / 6$ & $b r-f 03$ & psychoanalysis \\
\hline $\begin{array}{l}1252 \text { Energy, force and } \\
\text { power }\end{array}$ & 0.43 & $4 / 16$ & br-j07 & engineering/mechanics \\
\hline 22 American football & 0.41 & $6 / 18$ & br-a12 & American football \\
\hline $\begin{array}{l}748 \text { Churches, buildings } \\
\text { and organizations }\end{array}$ & 0.41 & $7 / 24$ & br-d03 & history of English church \\
\hline
\end{tabular}

Table 2: Mapping between CIDE+ categories and SEMCOR documents

\begin{tabular}{ccl}
\hline File & Word & Senses \\
\hline br-f03 & mind & head/brain (3), recall (1) \\
br-j07 & surface & outer boundary (1), extended 2D boundary (1) \\
br-a12 & football & game (4), object (1) \\
br-d03 & church & building (13), organisation (4), service (1) \\
\hline
\end{tabular}

Table 3: Senses from CIDE+ categories with more than sense in the identified SEMCOR file with number of occurrences in brackets

3 was 0 since, in these cases, the distribution was such that each type has a single sense with a probability of 1 and all others the probability 0 .

\subsection{Results}

In order to compare the various implemented methods with a naive approach a baseline was implemented. This randomly chose a sense from the set of possibilities for each word and assigned a probability of 1 to it and 0 to all other senses. The baseline was run 10 times and it was found that the mean of the average cross entropy scores over these 10 runs was 15.96 with a standard deviation of 1.83. The CIDE+ noun groups were also tagged manually with the annotator being asked to choose a single WordNet sense for each word in a group by considering the entire set of nouns it contains as evidence.
Like the automatic baseline calculation this was a forced choice task in which the annotator was asked to choose exactly one WordNet sense for each CIDE+ sense.

Table 4 shows the evaluation results for various metrics. It can be seen that all metrics perform better (lower ACE) than the random baseline. The best performance is observed from the product of all three metrics where the $\mathrm{ACE}$ obtained (5.76) is close to that obtained from the human annotator, representing a $94 \%$ reduction in error rate. This result is consistent with earlier work such as Stevenson and Wilks (2001) and McRoy (1992) which showed that word sense disambiguation is a task which benefits from a combination of multiple classifiers. Although this result should be considered in the context of the fact that a perfect match with the test data would return a score of 0.07 and 


\begin{tabular}{|rl|rl|}
\hline B Baseline & 15.96 & $\mathbf{6} I C+C D$ & 10.30 \\
$\mathbf{H}$ Human & 5.16 & $\mathbf{7} D O+I C+C D$ & 10.98 \\
$\mathbf{1 ~} D O$ & 11.93 & $\mathbf{8} D O * I C$ & 7.93 \\
$\mathbf{2} I C$ & 11.28 & $\mathbf{9} D O * C D$ & 5.80 \\
$\mathbf{3} C D$ & 9.31 & $\mathbf{1 0} I C * C D$ & 9.16 \\
$\mathbf{4} D O+I C$ & 11.86 & $\mathbf{1 1} D O * I C * C D$ & 5.76 \\
$\mathbf{5} D O+C D$ & 10.78 & & \\
\hline
\end{tabular}

Table 4: Results from various combinations of metrics

the manual annotation task is a forced choice of a single sense while the various metrics assign probabilities to senses.

It is interesting to note that there does not appear to be much difference between the additive combinations of metrics $(4-7)$ and the single metrics $(\mathbf{1}$ - 3). However, the products of metrics $(\mathbf{8}$ - 11) perform noticeably better. This may be because the multiplication combination is more conservative since all metrics must agree that there is some evidence for a particular sense. If any metrics assigns a zero probability to a sense then the product will be zero. Under these conditions each metric is acting as a filter and it appears that combining filters is a useful approach to this problem.

There is a noticeable difference between the performance of the $C D$ metric compared with the other two. This difference is not statistically significant according to a two-tail paired t-test although this may be due to the small amount of sample data. The pairwise correlations of the performance of each method on each of the 10 SEMCOR files was also quite high (between 0.79 and 0.89 ) indicating that some of the SEMCOR files were more difficult to disambiguate than others.

\section{Related Work}

Mandala et. al. (1999) combined three thesaurii to expand queries for an IR system. It was found that the combination of all three produced better results than no query expansion or when a single resource was used. The nature of their application meant that there was no need to produce an explicit mapping between the senses of the three resources.

Agirre et. al. (2000) constructed topic signatures constructed from web searches to add extra information to WordNet. A test set of 20 nouns which occur at least 100 times in
SEMCOR was chosen. For each possible WordNet sense a query was sent to the AltaVista search engine ${ }^{2}$ and the results stored. These documents were used to construct a topic signature for each concept which were evaluated within a sense disambiguation algorithm and found to outperform information extracted directly from WordNet. They produced further improvements when used to cluster senses.

Knight and Luk (1994) provided a mapping between WordNet and LDOCE by combining textual definitions with information about the hierarchical structure of the resources reporting 96\% mapping accuracy. Green et al. (2001) use a combination of similarity metrics, including Resnik's, to map entries in a verb database onto WordNet senses, reporting $72 \%$ precision and $58 \%$ recall. This suggests that the approach described here may be useful for other grammatical categories.

\section{Conclusion}

We have presented a method for overcoming the "tennis problem" in taxonomies such as WordNet by adding new relations to the hierarchy obtained by disambiguating the noun groups found in existing thesaural classes. It was found that this can be achieved using a combination of existing disambiguation techniques. The techniques were evaluated using gold standard taggings derived automatically from SEMCOR.

\section{Acknowledgements}

I am extremely grateful to Philip Resnik for advice on the re-implementation of the algorithm described in Section 2. This paper benefited from comments on earlier drafts provided by Yorick Wilks, George Miller and Tim Baldwin.

\footnotetext{
${ }^{2}$ http://www.altavista.com
} 
The work described here was carried out while the author was funded by the EPSRC grant "MALT: Mappings, Agglomerations and Lexical Tuning" (GR/M73521) and carried out in Sheffield University and while the author was on sabbatical at the Center for the Study of Language and Information, Stanford University.

\section{References}

E. Agirre and G. Rigau. 1996. Word sense disambiguation using conceptual density. In Proceedings of COLING '96, pages 16-22, Copenhagen, Denmark.

E. Agirre, O. Ansa, E. Hovy, and D. Martinez. 2000. Enriching very large ontologies using the WWW. In Proceedings of the ECAI Ontology Learning Workshop, pages 73-77, Berlin, Germany.

L. Burnard, 1995. Users Reference Guide for the British National Corpus. Oxford University Computing Services.

C. Fellbaum. 1998. WordNet: An Electronic Lexical Database and some of its Applications. MIT Press, Cambridge, MA.

W. Francis and H. Kucera. 1982. Frequency Analysis of English Usage. Hougton Mufflin Co., New York.

W. Gale, K. Church, and D. Yarowsky. 1992. One sense per discourse. In Proceedings of the DARPA Speech and Natural Language Workshop, pages 233-237, Harriman, NY.

R. Green, L. Pearl, B. Dorr, and P. Resnik. 2001. Mapping Lexical Entries in a Verb Database to WordNet Senses. In Proceedings of the 39th Annual Meeting of the Association for Computational Linguistics, pages 244-251, Toulouse, France.

S. Harabagiu and J. Chai, editors. 1998. Usage of WordNet in Natural Language Processing Systems. COLING-ACL-98 workshop. ACL.

K. Knight and S. Luk. 1994. Building a large knowledge base for machine translation. In Proceedings of the American Association for Artificial Intelligence Conference (AAAI-94), pages 185-109, Seattle, WA.

R. Krovetz. 1998. More than one sense per discourse. In Proceedings of SENSEVAL Workshop, Herstmonceux Castle, UK.

S. Landes, C. Leacock, and R. Tengi. 1998. Building a semantic concordance of English. In C. Fellbaum, editor, WordNet: An elec- tronic lexical database and some applications. MIT Press, Cambridge, MA.

M. Lesk. 1986. Automatic sense disambiguation using machine readable dictionaries: how to tell a pine cone from an ice cream cone. In Proceedings of ACM SIGDOC Conference, pages 24-26, Toronto, Canada.

R. Mandala, T. Tokunaga, and H. Tanaka. 1999. Combining general hand-made and automatically constructed thesauri for information retrieval. In Proceedings of the Sixteenth International Joint Conference on Artificial Intelligence (IJCAI-99), pages 920924, Stockholm, Sweden.

S. McRoy. 1992. Using multiple knowledge sources for word sense disambiguation. Computational Linguistics, 18(1):1-30.

P. Procter, editor. 1995. Cambridge International Dictionary of English. Cambridge University Press, Cambridge.

P. Resnik and D. Yarowsky. 1997. A perspective on word sense disambiguation techniques and their evaluation. In Proceedings of the SIGLEX Workshop "Tagging Text with Lexical Semantics: What, why and how?", pages 79-86, Washington, D.C.

P. Resnik. 1999. Disambiguating Noun Groupings with Respect to WordNet senses. In S. Armstrong, K. Church, P. Isabelle, S. Manzi, E. Tzoukermann, and D. Yarowsky, editors, Natural Language Processing using Very Large Corpora, pages 77-98. Kluwer Academic Press.

M. Stevenson and Y. Wilks. 2001. The interaction of knowledge sources in word sense disambiguation. Computational Linguistics, $27(3): 321-349$. 